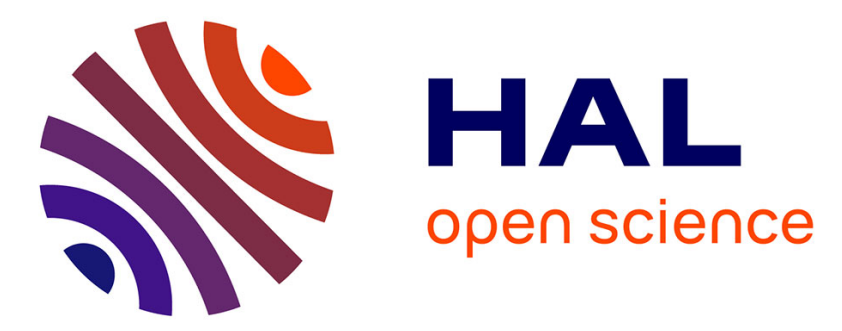

\title{
Mechanics of an adhesive tape in a zero degree peel test: effect of large deformation and material nonlinearity
}

Chung-Yuen Hui, Zezhou Liu, Helen Minsky, Costantino Creton, Matteo

Ciccotti

\section{- To cite this version:}

Chung-Yuen Hui, Zezhou Liu, Helen Minsky, Costantino Creton, Matteo Ciccotti. Mechanics of an adhesive tape in a zero degree peel test: effect of large deformation and material nonlinearity. Soft Matter, 2018, 14 (47), pp.9681-9692. 10.1039/C8SM01731J . hal-02076946

\section{HAL Id: hal-02076946 https://hal.science/hal-02076946}

Submitted on 3 May 2019

HAL is a multi-disciplinary open access archive for the deposit and dissemination of scientific research documents, whether they are published or not. The documents may come from teaching and research institutions in France or abroad, or from public or private research centers.
L'archive ouverte pluridisciplinaire HAL, est destinée au dépôt et à la diffusion de documents scientifiques de niveau recherche, publiés ou non, émanant des établissements d'enseignement et de recherche français ou étrangers, des laboratoires publics ou privés. 


\title{
Mechanics of an adhesive tape in a zero degree peel test: effect of large deformation and material nonlinearity $\dagger$
}

\author{
Chung-Yuen Hui, * *a Zezhou Liu, a Helen Minsky, b Costantino Creton b \\ and Matteo Ciccotti b
}

\begin{abstract}
The common pressure sensitive adhesive (PSA) tape is a composite consisting of a stiff backing layer and a soft adhesive layer. A simple and common way to test how adhesive tapes respond to large shear deformations is the zero degree peel test. Because the backing is very stiff compared to the adhesive layer, the region where the adhesive layer is subjected to large shear can be hundreds of times its thickness. We use a large deformation hyperelastic model to study the stress and deformation fields in the adhesive layer in this test. We present a closed-form solution for the stress field in the adhesive layer and use this solution to determine how load is transferred from the backing layer to the adhesive. Our analytical model is then compared with finite element results, and except for a small region near the peel front, the predicted stress and deformation agree well with the finite element model. Interestingly, we find very different results from the classical linear theory established by Kaelble. In particular for large deformations, our analysis shows that the lateral stresses (parallel to the rigid substrate) are much larger than the shear stress in the adhesive layer. The discrepancy in the stress state and the deformation state with the linear theory is particularly large near the peel front, which we study with a finite element model. These new results will be very useful to interpret experiments and in particular to identify the high stress regions where failure is likely to initiate in zero-degree peel tests also called shear resistance tests in the PSA industry.
\end{abstract}

\section{Introduction}

A common way of studying the adhesive properties of pressure sensitive adhesives (PSAs) is the tack test, where a flat steel indenter is retracted after being pressed in contact with the surface of a thin adhesive layer. ${ }^{2-6}$ The force versus indenter displacement curve in this test provides important insights into the failure mechanism under predominantly hydrostatic tension. ${ }^{7,8}$ However, in applications, PSAs are often used as lap joints where shear deformation is dominant. In industry, the standard test for shear strength is to conduct a loadcontrolled zero degree peel test ${ }^{9,10}$ to measure the time needed to detach a standardized area of the adhesive tape from a stiff substrate under a fixed moderate load (e.g. by a hanging weight, schematics shown in Fig. 1). ${ }^{11,12}$ Alternatively PSA can be debonded in shear in displacement controlled mode as for (a)

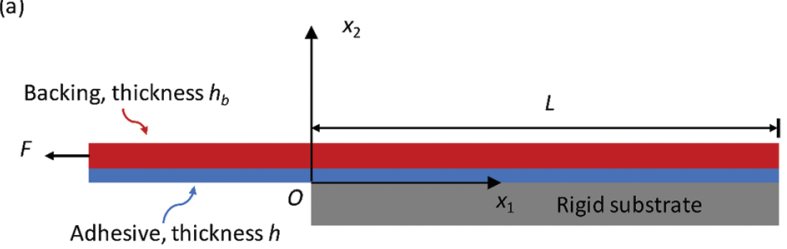

(b)

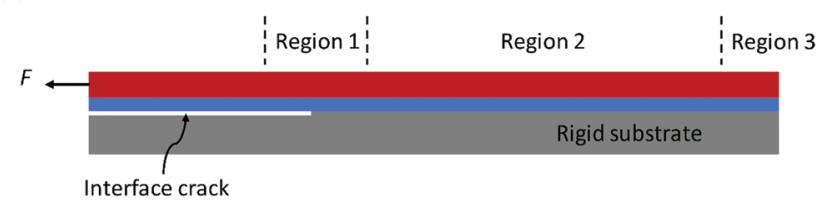

Fig. 1 Schematics of a zero degree peel test. (a) $L$ is the bond length and is assumed to be infinite in extent. The adhesive and backing thicknesses are represented as $h$ and $h_{\mathrm{b}}$ respectively. The applied force $F$ is usually provided by a hanging weight. (b) The overhanging adhesive surface up to the peel front $\left(x_{1}<0, x_{2}=0\right)$ can be seen as an interface crack. Region 1 is close to the peel front, and region 2 occupies the rest of the bond length except a small region near the right edge, which we named region 3 . In this work, region 3 does not exist since $L$ is considered to be infinite. 
example proposed by Sosson et $a .^{13}$ In this method, a soft thin adhesive layer is sheared between a hemispherical glass indenter and a flat glass substrate. Nevertheless, the standard load controlled test is widely accepted and commonly used because of its practical relevance.

There is a large body of literature concerned with the mechanics of the peel adhesion test including peeling of heterogeneous films ${ }^{14}$ and failure of lap shear joints, ${ }^{15}$ and here we focus on the zero degree peel test. A well-known analysis of the peel test for all peel angles was given by Kaelble in $1960 .^{1}$ In his analysis, the backing layer is modeled as an elastic plate. Since the deformation of the backing layer is assumed to be small, the bending and in-plane stresses are decoupled. The tension in the backing layer is coupled to the shear stress of the adhesive layer by the shear lag model. ${ }^{16,17}$ Kaelble's result for the zero degree peel test will be discussed in more detail below. More recently, the zero degree peel test has been studied by several research groups, with different emphasis. For example, Ponce et al. ${ }^{18}$ and Collino et al. ${ }^{19}$ studied the effect of interfacial friction on peeling. The mechanics of their analysis is based on a simplified version of Kaelble's peel model - they use a shear lag model to study the shear stress in the adhesive layer and ignore the bending of the backing layer which induces a normal stress concentration at the peel front. Cohen $e t a l .{ }^{20}$ studied failure of a thin adhesive pad under loading parallel to the substrate. Mojdehi et $a l .{ }^{17}$ used the same shear-lag approach to study the effect of compliance on the fracture mechanics of the zero degree peel test. Their analysis reconfirms the classical fracture mechanics' result that the compliance of the load strain should not affect the bond strength, whereas if the area-to-compliance ratio is used as a fracture parameter, it directly influences the bond strength. ${ }^{18,21-25}$

Two key assumptions in the standard analysis of the zero degree peel adhesion test require further study. The first assumption is that the deformation of the adhesive layer is sufficiently small so that it can be modeled as a linear elastic solid. However, in practice, PSAs are only lightly cross-linked to provide creep resistance and they are thus very soft. ${ }^{26}$ A typical nominal stress versus stretch behavior is shown in Fig. 2. For small strains, the curve is linear; at larger strains, the material softens, as indicated by a decrease in tangent modulus; finally, at high strains, the adhesive hardens. Although extensive investigations of unloading of PSAs have rarely been published, most of the deformation is reversible and elastic although it is strain rate dependent. ${ }^{27,28}$

Given that in a zero degree peel test, the nominal shear strain of the adhesive layer can become as high as $1000 \%$, the assumption of linear elasticity cannot possibly hold. In this work, we will show that large deformation induces a lateral stress (parallel to the rigid substrate) that is much larger than the shear stress in the adhesive.

Local failure mechanisms (cohesive failure or interfacial failure or a mixture of both, cavitation or interfacial debonding) are sensitive to the stress state at the peel front and this sensitivity brings up another key assumption: that is, the use of a structural mechanics model to analyze the stress and deformation states near the peel front. Since the peel front is

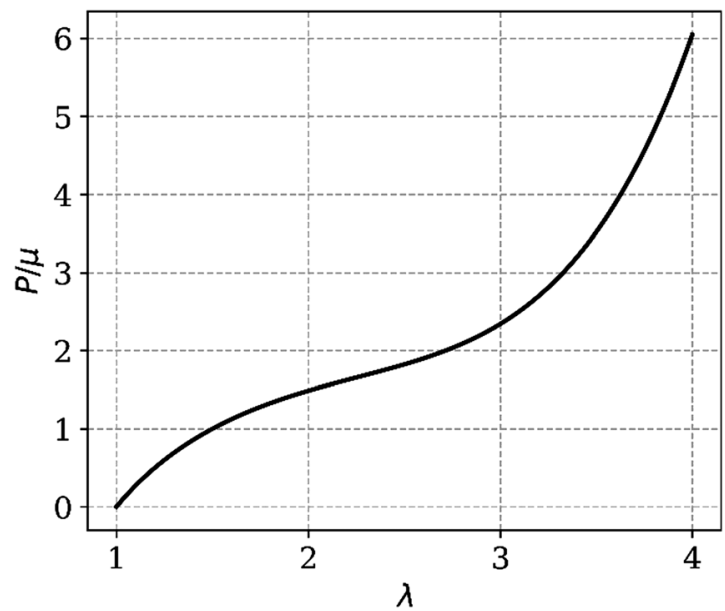

Fig. 2 Nominal stress $P$ (normalizes by the shear modulus $\mu$ ) versus stretch ratio $\lambda$ for Yeoh's material with three terms $C_{1}=0.5 \mu, C_{2}=-0.0237 \mu$ and $C_{3}=0.00166 \mu$.

the tip of an interface crack, the stress state is highly concentrated and three dimensional. For example, if the adhesive was modeled as linearly elastic, then in a full 3D analysis the stress will have an inverse square root singularity as the peel front is approached. ${ }^{29}$ The peel test for a zero thickness adhesive layer using the fracture mechanics approach was studied in detail by Thouless and Yang. ${ }^{30}$ In their analysis, the peel front is a mixed mode crack characterized by mode I and mode II stress intensity factors, which can be related to the applied peel force for a given peel angle. However, their results are not directly applicable to PSAs because (1) they assume small strain linear elasticity; (2) their adhesive layer thickness is zero, whereas in our case the adhesive layer thickness is comparable to the backing layer thickness. A specific difficulty is that in the linear theory, the mode-I stress intensity factor becomes negative as the peel angle is reduced to zero. It is difficult to imagine how the interface can fail under infinite compression. In summary, a plate model (linear or nonlinear) cannot accurately capture the stress state at the peel front. Finally, most structural models in the literature assume that the stress and deformation in the adhesive layer are independent of the thickness coordinate $x_{2}$ in Fig. 1). Although this assumption is reasonable at distances far away from the peel front, it breaks down as the peel front is approached. Indeed, our analysis shows that the normal stress on the interface between the adhesive/substrate and the adhesive/backing interface can have the opposite sign.

The goal of this study is to develop a model for the zero degree peel test without the usual assumption of small strains and linear elasticity. Thus, the adhesive can sustain arbitrarily large deformations. In addition, our material model also captures the type of nonlinear elastic behavior that is exhibited by PSAs. Our focus is on adhesive tapes with backing much stiffer than the adhesive. This feature implies that the region of the tape that is supporting shear can be much greater than the thickness of the adhesive layer. In this work we assume that the bond length $L$ is infinite, that is, it is long in comparison with the load transfer length. Fig. 1b shows that the tape can be 
divided into three regions: region 1 is close to the peel front, where there is a large stress concentration and where a nonlinear finite element (FE) analysis is necessary. The length of region 1 is on the order of several adhesive layer thicknesses (this will be confirmed by FE analysis). Region 2 occupies the rest of the bonded tape, except for a small region near the right edge, which we called region 3. In this work, region 3 does not exist since $L$ is considered to be infinite. The stresses in region 2 can still be very large, and are not necessarily dominated by shear. We determine the stresses and strains in this region in a closed form using a combination of asymptotic analysis and a nonlinear shear lag model. Our result is then verified using a nonlinear finite element model (FEM).

\section{Model and geometry}

The adhesive tape is modeled as a composite consisting of a stiff backing bonded to the soft adhesive ( $c f$. Fig. 1). The thickness of the backing and the adhesive layer are assumed to be uniform and denoted by $h_{\mathrm{b}}$ and $h$ respectively. A constant force $F$ is applied to the tape at one end (e.g. by a hanging weight). A segment of the tape, of length $L$, is adhered to a flat glass substrate, whereas the rest of the tape hangs over the edge. In practice, the bond length $L$ can be thousands of times longer than the tape thickness $h+h_{\mathrm{b}}$.

The left bonded edge of the substrate coincides with the origin of a fixed coordinate system $\left(x_{1}, x_{2}\right)$. The portion of tape in contact with the substrate occupies $x_{1} \in[0, L]$. The peel arm is the tape that is not in contact with the substrate. Since the width of the tape $b$ is much greater than its thickness, we assume plane strain deformation where the out-of-plane displacement $u_{3}$ is zero and the in-plane displacements $u_{\alpha}$ $(\alpha=1,2)$ depend only on the in-plane coordinates $\left(x_{1}, x_{2}\right)$. Since the applied force is uniformly distributed across its width, the appropriate force measured in a plane strain analysis is $F / b$ which has the unit of force per unit length. Before load is applied, the tape is assumed to be stress free and a material point in the tape is identified by its coordinates $\left(x_{1}, x_{2}\right)$. After load is applied, the material point occupies $\left(y_{1}, y_{2}\right)$ with respect to the same coordinate system, thus $y_{\alpha}=x_{\alpha}+u_{\alpha}$.

To motivate our model, we note that the typical shear modulus of a pressure-sensitive-adhesive is less than $0.1 \mathrm{MPa}$, whereas the Young's modulus of the backing layer is on the order of a few GPa. ${ }^{31}$ This large difference results in a very long portion of the adhesive that is carrying load, which is named the load transfer length $l_{\mathrm{LT}}\left(l_{\mathrm{LT}}=\infty\right.$ for a backing layer that is infinitely stiff). Further, since the modulus of the substrate is even larger than the backing, it is modeled as rigid. For a good general purpose adhesive such as an office tape, the nominal shear strain of the adhesive layer can approach or exceed 1000 percent before slip or failure occurs, ${ }^{32-35}$ so any realistic model of this layer has to consider large deformation.

The contact region $[0, L]$ can be divided into three distinct regions. Since the substrate is rigid, the peel front at $x_{1}=x_{2}=0$ can be viewed as the tip of an interface crack (see Fig. 1b). Near this edge, the stresses and deformation are dominated by the presence of the crack. This region of high stress is called region 1 and occupies $x_{1} \in(-d, d)$, where $d$ is on the order of the tape thickness. Likewise, the right edge at $x_{1}=L, x_{2}=0$ is a corner, but since the bond length is very long the load transmitted to this corner is small; furthermore, the strength of the singularity at the corner is lower than that at the crack tip (region 3 consists of the material near this corner). In a typical test, $L \geq l_{\mathrm{LT}} \gg h$, so most of the tape that is in contact occupies the region in between the two edges (region 2). However, since the adhesive layer is very thin in comparison to $l_{\mathrm{LT}}$, the adhesive within $x_{1} \in\left(d, l_{\mathrm{LT}}\right)$ can be subjected to very large shear strain, on the order of $1000 \%$. In most models of zero degree peel, the adhesive in this region is assumed to be under a state of pure shear stress, where other stress components are neglected since they are assumed to be small. Although this approximation is valid for small deformation, it breaks down when the deformation is large, as we will demonstrate below. In the following, we shall assume $L \gg l_{\mathrm{LT}}\left(L / l_{\mathrm{LT}} \rightarrow \infty\right)$ so we can ignore the right edge or region 3 in our analysis.

The nominal stress $P$ versus stretch ratio $\lambda$ of a typical adhesive in a uniaxial test can be found in Chopin et al. ${ }^{32}$ and Deplace et al., ${ }^{36}$ and is represented sufficiently well by a three-term Yeoh's hyperelastic model in Fig. 2. It should be noted that there is no universally accepted model for the constitutive behavior of adhesives, here we use the simplest model that can capture many of the key features observed in a tensile test. $^{36}$ These are: for small stretch ratios, the stress versus stretch is approximately linear; the slope of the stress versus stretch curve decreases (softening) at immediate stretches; at large stretches the stress increases rapidly as the adhesive strain hardens.

The strain energy density function $\Phi$ of Yeoh's material has the form:

$$
\Phi=\sum_{k=1}^{N} C_{k}\left(I_{1}-3\right)^{k}
$$

where $N$ is the number of terms, $C_{k}$ are the material constants with units of stress and $I_{1}$ is the trace of the right Cauchy-Green tensor, i.e.,

$$
I_{1}=\operatorname{tr}\left(\mathbf{F}^{\mathrm{T}} \mathbf{F}\right)
$$

where $\mathbf{F}$ is the deformation gradient tensor and the superscript $\mathrm{T}$ denotes its transpose. Here the material is assumed to be incompressible. In particular, $2 C_{1}=\mu$ is the small strain shear modulus. Note that for $C_{2}=C_{3}=0$, Yeoh's model reduces to the neo-Hookean model. However, the normalized nominal stress of a neo-Hookean material in a uniaxial test does not exhibit softening and hardening behaviors (see the ESI $\dagger$ for details), and hence is not expected to capture real adhesive behavior such as those studied in Chopin et al. ${ }^{32}$ or Deplace et al. ${ }^{36}$

\subsection{Load transfer length $l_{\mathrm{LT}}$}

The variation of shear stresses along the adhesive layer can be modeled using the well-known shear-lag model. Denote the 
shear stress on the interface between the backing and the adhesive by $\tau$ and the horizontal displacement of the backing layer by $u_{\mathrm{b}}\left(x_{1}\right)$, i.e., it is uniform in the thickness direction. In the shear-lag model, $\tau$ is in general a nonlinear function of the average shear strain $\bar{\gamma} \equiv u_{\mathrm{b}} / h$. Here we have used the usual assumption that the backing layer is under uniaxial tension and $u_{\mathrm{b}}\left(x_{1}\right)=u\left(x_{1}, x_{2}=h\right)$, that is, displacements are continuous across the backing/adhesive interface. In the shear-lag model,

$$
\frac{E_{\mathrm{b}}{ }^{*} h_{\mathrm{b}} h}{\mu} \frac{\mathrm{d}^{2} \bar{\gamma}}{\mathrm{d} x_{1}{ }^{2}}=\hat{\tau}(\bar{\gamma}),
$$

where $E_{\mathrm{b}}{ }^{*}$ is the plane strain modulus of the backing and $\hat{\tau}(\bar{\gamma}) \equiv$ $\tau(\bar{\gamma}) / \mu$ is the normalized shear stress. The quantity

$$
l_{\mathrm{LT}} \equiv \sqrt{E_{\mathrm{b}}{ }^{*} h_{\mathrm{b}} h / \mu}
$$

is defined as the load transfer length. Indeed, if we normalize distance by

$$
\eta=x_{1} / l_{\mathrm{LT}}
$$

then (2) becomes

$$
\frac{\mathrm{d}^{2} \bar{\gamma}}{\mathrm{d} \eta^{2}}=\hat{\tau}(\bar{\gamma}) .
$$

Note $l_{\mathrm{LT}} / h=\sqrt{E_{\mathrm{b}}{ }^{*} h_{\mathrm{b}} / \mu h}$ and since $h_{\mathrm{b}}$ is typically on the same order as $h, l_{\mathrm{LT}} / h \approx \sqrt{E_{\mathrm{b}}{ }^{*} / \mu}$. This means that the load transfer length $l_{\mathrm{LT}}$ is hundreds of times the adhesive thickness. Note that in order to use the shear-lag model, we have assumed that the normal traction on the backing/adhesive interface is zero, and this will be justified below.

\subsection{Analysis of region 2}

We first give an approximate analysis of the stress state in region 2. Our analysis is verified by a nonlinear finite element model. Our idea is based on the fact that the modulus of the backing layer is at least 4 orders of magnitude higher than the adhesive, as a result, the variation of stress/deformation along the $x_{1}$ direction must be very slow. Hence the local stress versus strain relation can be determined by assuming that the backing layer is rigid (this will be verified below). Our analysis below is based on finite strain theory.

The symmetric true stress tensor $\tau$ in the adhesive layer is related to the deformation gradient tensor $\mathbf{F}$ by

$$
\tau=-p \mathrm{I}+2 \frac{\mathrm{d} \Phi}{\mathrm{d} I_{1}} \mathbf{B}
$$

where $\mathbf{B}=\mathbf{F F}^{\mathrm{T}}$ is the left Cauchy-Green tensor, $p$ is the Lagrange multiplier or pressure needed to enforce incompressibility and $\mathbf{I}$ is the identity tensor. In plane strain, $\mathbf{F}$ is:

$$
\mathbf{F}=\left[\begin{array}{ccc}
1+u_{1,1} & u_{1,2} & 0 \\
u_{2,1} & 1+u_{2,2} & 0 \\
0 & 0 & 1
\end{array}\right]
$$

where $u_{\alpha, \omega} \equiv \partial u_{\alpha} / \partial x_{\omega}(\alpha, \omega=1,2)$. The in-plane components of $\mathbf{B}$ can be calculated using (7), they are:

$$
B_{11}=\left(1+u_{1,1}\right)^{2}+\left(u_{1,2}\right)^{2}>0,
$$

$$
\begin{gathered}
B_{12}=B_{21}=\left(1+u_{1,1}\right) u_{2,1}+\left(1+u_{2,2}\right) u_{1,2}, \\
B_{22}=\left(u_{2,1}\right)^{2}+\left(1+u_{2,2}\right)^{2},
\end{gathered}
$$

Also, $I_{1}$ is

$$
I_{1}=\left(1+u_{1,1}\right)^{2}+\left(u_{2,1}\right)^{2}+\left(1+u_{2,2}\right)^{2}+\left(u_{1,2}\right)^{2}+1 .
$$

Define the shear strain $\gamma \equiv u_{1,2}$ (which corresponds to the average shear strain $\bar{\gamma}$ in the previous section). For large shear strain, $\gamma \gg 1$, we assume

$$
\left|u_{2,1}\right|, \quad\left|u_{1,1}\right| \quad\left|u_{2,2}\right| \ll 1 \ll \gamma .
$$

Eqn (8) and (10) imply that

$$
\begin{gathered}
B_{11}=\left(1+u_{1,1}\right)^{2}+\left(u_{1,2}\right)^{2} \approx \gamma^{2} \\
B_{12}=\left(1+u_{1,1}\right) u_{2,1}+\left(1+u_{2,2}\right) u_{1,2} \approx \gamma\left(1+u_{2,2}\right) .
\end{gathered}
$$

Since the material is incompressible,

$$
\operatorname{det} \mathbf{B}=\operatorname{det}\left(\mathbf{F F}^{\mathrm{T}}\right)=\operatorname{det} \mathbf{F d e t} \mathbf{F}^{\mathrm{T}}=1 \Rightarrow B_{11} B_{22}-\left(B_{12}\right)^{2}=1 .
$$

Eqn (12) implies that

$$
B_{22}=\frac{\left(B_{12}\right)^{2}+1}{B_{11}} \text {. }
$$

Substituting (11a) and (11b) into (13a) results in

$$
B_{22} \approx\left(1+u_{2,2}\right)^{2} \text {. }
$$

Substituting (13b) and (11a, b) into (12), the incompressibility condition (12) can be written as:

$$
u_{1,1}+u_{2,2} \approx 0 \text {. }
$$

consistent with our expectations that all normal strains are small as long as the adhesive is well-bonded to the glass substrate. Since the upper surface of the backing layer is exposed to air, we expect the normal stress $\tau_{22}$ to vanish inside region 2 . By (6), this condition requires

$$
p=\mu f\left(I_{1}\right) B_{22}
$$

where $\mu f\left(I_{1}\right) \equiv 2 \mathrm{~d} \Phi / \mathrm{d} I_{1}$. Substituting (15a) into (6), we find

$$
\tau_{11}=\mu f\left(I_{1}\right)\left(B_{11}-B_{22}\right) .
$$

Substituting (13b) and (11a) into (15b), we find

$$
\tau_{11} \approx \mu f\left(I_{1}\right) \gamma^{2} .
$$

Finally, (6) and (11b) imply that

$$
\tau_{12}=\mu f\left(I_{1}\right) B_{12} \approx \mu f\left(I_{1}\right) \gamma .
$$

Note

$$
I_{1}-3 \approx \gamma^{2}
$$

so $f\left(I_{1}\right)$ is a function of the shear strain $\gamma$.

Our analysis expresses all the true stress components as functions of the shear strain. Eqn (15c) and (15d) show that, for large shear strains, the dominant stress is $\tau_{11}-\operatorname{not} \tau_{12}$. 
Indeed, the ratio of $\tau_{12} / \tau_{11} \approx \gamma^{-1}$ vanishes for large shear strain. Thus, the order of the in-plane true stress is: $\left|\tau_{11}\right| \gg\left|\tau_{12}\right| \gg$ $\left|\tau_{22}\right| \approx 0$. Note that the equilibrium equations are satisfied if the shear strain is independent of the position, i.e., the backing layer is rigid.

\subsection{Relation between zero degree peel force and stress in the adhesive layer}

Having established that the local true stresses are related to the strains by the simple relationships (15c) and (15d), we are in a position to study load diffusion using the shear lag model. This will allow us to relate the shear strain $\gamma$ to the peel force. Eqn (5) is nonlinear, but it can be solved exactly by making $\gamma$ the independent variable and $\eta$ the dependent variable. An exact solution can be found for any incompressible hyperelastic model where the strain energy density function depends only on $I_{1}$. Here we report the result for the special case of Yeoh's material where $f\left(I_{1}\right)=\left(1+\frac{4 C_{2}}{\mu} \gamma^{2}+\frac{6 C_{3}}{\mu} \gamma^{4}\right)$ :

$$
\eta(\gamma)=\frac{x_{1}}{l_{\mathrm{LT}}}=\ln \left|\frac{\gamma_{0}}{\gamma}\right|-\frac{1}{2} \ln \left[\frac{\sqrt{1+\frac{2 C_{2}}{\mu} \gamma_{0}^{2}+\frac{2 C_{3}}{\mu} \gamma_{0}^{4}}+\frac{C_{2}}{\mu} \gamma_{0}^{2}+1}{\sqrt{1+\frac{2 C_{2}}{\mu} \gamma^{2}+\frac{2 C_{3}}{\mu} \gamma^{4}}+\frac{C_{2}}{\mu} \gamma^{2}+1}\right],
$$

where $\gamma_{0}$ is the as yet unknown maximum shear strain at the origin. Details of the derivation can be found in the ESI. $\dagger$ Eqn (16a) dictates how the shear strain in the adhesive layer varies with position. Eqn (16a) can be solved to yield the shear strain $\gamma$ as a function of normalized position $\eta=x_{1} / l_{\mathrm{LT}}$, i.e.,

$$
\gamma^{2}=\frac{2 \mathrm{e}^{-2 \eta}}{A\left(\gamma_{0}\right)\left\{1-\frac{2 C_{2}}{\mu A\left(\gamma_{0}\right)} \mathrm{e}^{-2 \eta}+\frac{\mathrm{e}^{-4 \eta}}{A^{2}\left(\gamma_{0}\right)}\left[\left(\frac{C_{2}}{\mu}\right)^{2}-\frac{2 C_{3}}{\mu}\right]\right\}},
$$

where $A\left(\gamma_{0}\right) \equiv \sqrt{\gamma_{0}{ }^{-4}+\frac{2 C_{2}}{\mu} \gamma_{0}{ }^{-2}+\frac{2 C_{3}}{\mu}}+\frac{C_{2}}{\mu}+\gamma_{0}{ }^{-2}$ (see the ESI $\dagger$ ). Note that the shear strain decays exponentially fast with characteristic length equal to the load transfer length. Since the stresses are related to the shear strains by (15c) and (15d), they also decay exponentially fast away from the peel front.

The maximum shear strain $\gamma_{0}$ can be related to the peel force $F / b$, which is the integral of the shear stress along the adhesive/ backing interface. The derivation of this relation is given in the ESI, $\uparrow$ for a three-term Yeoh's solid, it is

$$
F / b=-\mu \underbrace{\sqrt{\frac{E_{\mathrm{b}}{ }^{*} h_{\mathrm{b}}}{\mu h}}}_{l_{\mathrm{LT}}}\left|\gamma_{0}\right| \sqrt{1+\frac{2 C_{2}}{\mu}\left|\gamma_{0}\right|^{2}+\frac{2 C_{3}}{\mu}\left|\gamma_{0}\right|^{4}} .
$$

The force is negative since it points in the negative $x_{1}$ direction. Eqn (17) can be solved exactly to determine $\gamma_{0}$ in terms of $F$, and the solution is: ${ }^{37}$

$$
\left|\gamma_{0}\right|=\sqrt{s_{1}+s_{2}-\frac{C_{2}}{3 C_{3}}}
$$

where

$$
\begin{gathered}
s_{1}=\left(r+\sqrt{r^{2}+q^{3}}\right)^{1 / 3}, \\
s_{2}=\left(r-\sqrt{r^{2}+q^{3}}\right)^{1 / 3}, \\
r=\frac{\mu}{4 C_{3}}\left[\frac{C_{2}}{3 C_{3}}+\frac{(F / b)^{2}}{\mu^{2} l_{\mathrm{LT}^{2}}}\right]-\left(\frac{C_{2}}{3 C_{3}}\right)^{3}, \\
q=\frac{\mu}{6 C_{3}}-\left(\frac{C_{2}}{3 C_{3}}\right)^{2} .
\end{gathered}
$$

Eqn (18a)-(18e) show that a nonlinear relation exists between the maximum shear strain and the peel force. Finally, the shear stress $\tau_{12}$ and the lateral normal stress $\tau_{11}$ in the adhesive layer can be obtained in a closed form by substituting (16a), (16b) and (18a)-(18e) into (15c) and (15d). In the limit of small strains, the solution mentioned above reduces to the result of Kaelble ${ }^{1}$ by formally setting $C_{2}=C_{3}=0$. For this case, the maximum shear strain $\gamma_{0}$ is directly proportional to the force. Fig. 3 plots the maximum shear strain $\gamma_{0}$ versus the normalized peel force $\bar{F} \equiv(F / b) / \mu l_{\text {LT }}$ determined by eqn (17). The linear theory of Kaelble is also shown in the same figure (dashed line) for comparison. For large deformation, the linear theory considerably underestimates the force at the same strain $\gamma_{0}$ due to strain hardening. It is interesting to note that the linear theory overestimates the force for shear strains between -2 and -4 , and this is due to the effect of strain softening. We emphasize that the qualitative behavior of the nonlinear solution holds regardless of the choice of parameters chosen for the Yeoh model. In the ESI, $\dagger$ we plot two other sets of parameters to justify this statement.

Fig. 4 plots the maximum normalized shear $\bar{\tau}_{12}^{\max } \equiv \tau_{12}^{\max } / \mu$ and the normal stress $\bar{\tau}_{11}^{\max } \equiv \tau_{11}^{\max } / \mu$ against the normalized peel force (solid lines). The linear theory of Kaelble is plotted on the same figure (dashed lines) for comparison. For the shear stress,

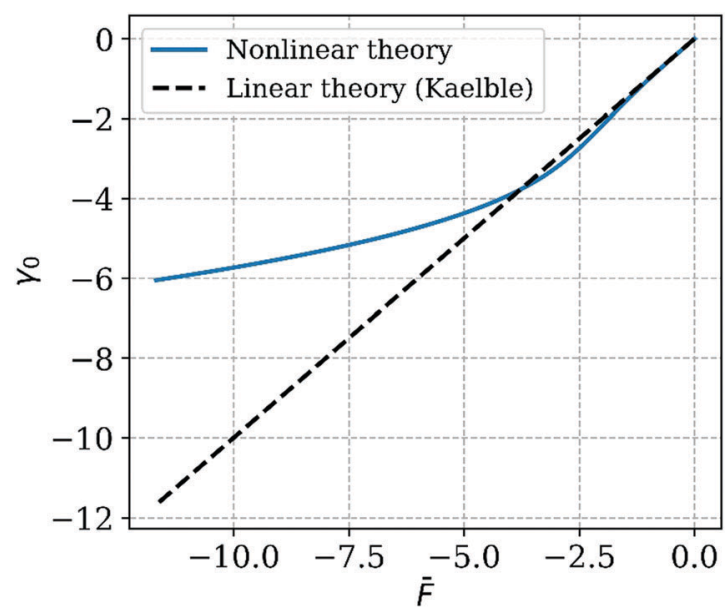

Fig. 3 Normalized peel force $\bar{F}$ versus maximum shear strain $\gamma_{0}$ at the peel front. Nonlinear theory and linear theory (Kaelble) predictions, for various $\bar{F}$, are plotted as solid and dashed lines respectively. 
the linear theory predicts a slope of one. However, the nonlinear theory shows that the maximum shear stresses increase faster with increasing peel force. Indeed, eqn (17) shows that in the limit where the shear strain is sufficiently large so that $1+\frac{2 C_{2}}{\mu}\left|\gamma_{0}\right|^{2}+\frac{2 C_{3}}{\mu}\left|\gamma_{0}\right|^{4} \approx \frac{2 C_{3}}{\mu}\left|\gamma_{0}\right|^{4}$, that is, when strain hardening dominates, $F / b \approx-\mu l_{\mathrm{LT}} \sqrt{\frac{2 C_{3}}{\mu}}\left|\gamma_{0}\right|^{3}$. In particular, note that the linear theory assumes $\tau_{11}=0$, whereas the lateral normal stress is much larger than the shear stress at large deformation. This conclusion is always valid and is independent of the hyperelastic model. Indeed, equations ((15c) and (15d)) indicate that $\tau_{11} \approx \tau_{12} \gamma$ for large shear strain.

\section{Finite element (FE) analysis}

The finite element model and the mesh strategy are shown schematically in Fig. 5 and implemented in a commercial FEM software, ABAQUS ${ }^{\circledR}$. The adhesive is modeled by a three-term Yeoh's material with $C_{1} / \mu=0.5, C_{2} / \mu=-0.0237$ and $C_{3} / \mu=$ 0.00166 , where $\mu=2 C_{1}$ is the small strain shear modulus (Fig. 2). The backing layer is modeled as a linear elastic solid with Young's modulus, $E_{\mathrm{b}}=30000 \mu$, and Poisson's ratio, $\nu=0.3$. In the simulation, the overlap length $L=1000 h$ is approximately 5 times the load transfer length $l_{\mathrm{LT}}$, and the overhanging length is $20 h$. The initial thickness of the backing $h_{\mathrm{b}}$ is considered to be $2 h$ (which is representative of typical tapes ${ }^{31}$ ). In this FE model, we normalize all distances by the initial thickness of the adhesive $h$ and all stress quantities by the small strain shear modulus of the adhesive, $\mu$.

The displacements on the adhesive/substrate interface are fixed to be zero. The constant force $F / b$ is mimicked by a horizontal traction $t$ acting on the left end of the backing, and the integral of this traction over the backing's end in the current configuration is maintained to be equal to $F / b$. To balance the accuracy and efficiency of the computation, we choose a very fine mesh near the origin in the adhesive, while far away the

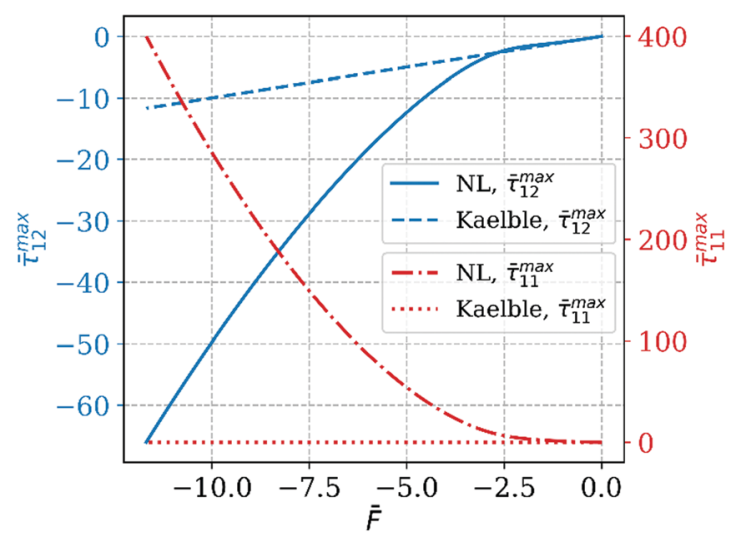

Fig. 4 Normalized peel force $\bar{F}$ versus maximum normalized shear $\bar{\tau}_{12}^{\max }$ (solid blue line) and lateral normal stress $\bar{\tau}_{11}^{\max }$ (dashed-dotted red line). The linear theory of Kaelble is plotted as dashed and dotted lines. Note that the linear theory assumes $\tau_{11}=0$.

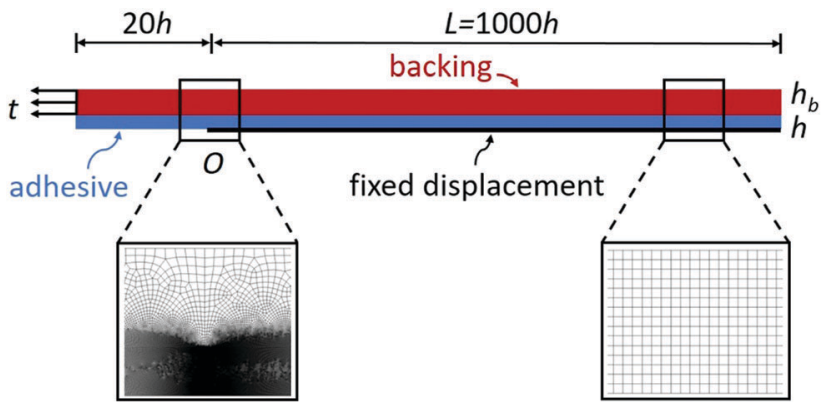

Fig. 5 Schematic of a finite element model and mesh strategy. The red and blue filled rectangles represent the backing layer and adhesive layer respectively, and the rigid substrate is modeled by the fixed displacement boundary condition.

element size increases and is about $0.2 h$ near the end. Hybrid plane strain elements CPE4H and CPE3H are used to simulate the incompressibility of the adhesive. Our convergence test shows that further refinement of the mesh does not affect the FE results (except the first few elements near the origin where there is a singularity).

\subsection{Comparison between theory and a FEM}

For a given force $F,(16)$ and (18) allow us to obtain the shear strain distribution along the adhesive/backing interface. To compare with the FEM result, we set

$$
\gamma=\frac{u_{1}^{\mathrm{FE}}\left(\bar{x}_{1}, \bar{x}_{2}=1\right)}{h},
$$

where $\bar{x}_{1}=x_{1} / h, \bar{x}_{2}=x_{2} / h$ and $u_{1}^{\mathrm{FE}}\left(\bar{x}_{1}, \bar{x}_{2}=1\right)$ is the horizontal displacement at the adhesive/backing interface extracted from the FEM results. Fig. 6 plots the shear strain $\gamma$ as a function of the normalized position $\bar{x}_{1}$. The solid lines are the analytic solutions given by eqn (16) and (18), and the FEM results are shown as symbols for different values of normalized peel force $\bar{F}$. This comparison demonstrates that our analytic solution matches the FEM results extremely well. Also, Fig. 6 shows the exponential decay in the shear strain distribution.

In our analytical model, the stresses in the adhesive layer are assumed to be approximately independent of $x_{2}$. Eqn (16), (18) and (15d) allow us to express the interfacial true shear stress between the adhesive layer and the substrate as a function of normalized position $\bar{x}_{1}$; these results are plotted in Fig. 7 for different values of the normalized force $\bar{F}$. The finite element results (true stress, symbols) are also plotted. In order to compare results with different peel forces in one plot, we divide $\bar{\tau}_{12}$ and $\bar{\tau}_{11}$ by $\bar{\tau}_{12}^{\max }$ and $\bar{\tau}_{11}^{\max }$ respectively, where $\bar{\tau}_{12}^{\max }$ and $\bar{\tau}_{11}^{\max }$ are the maximum values of these normalized stresses evaluated at the origin using our analytic solution (see Fig. 4). Here it is important to remember that $\bar{\tau}_{12}^{\max }$ and $\bar{\tau}_{11}^{\max }$ depend on the peel force nonlinearly (see Fig. 4), hence the actual stresses increase rapidly with the peel force. Again, the finite element result agrees very well with the analytical model as long as $\bar{x}_{1}$ is in region 2. The insets in Fig. 7a-c show that our analytical model breaks down in a region about 3 times the thickness of the adhesive tape. Indeed, our result shows that the analytical 
(a)

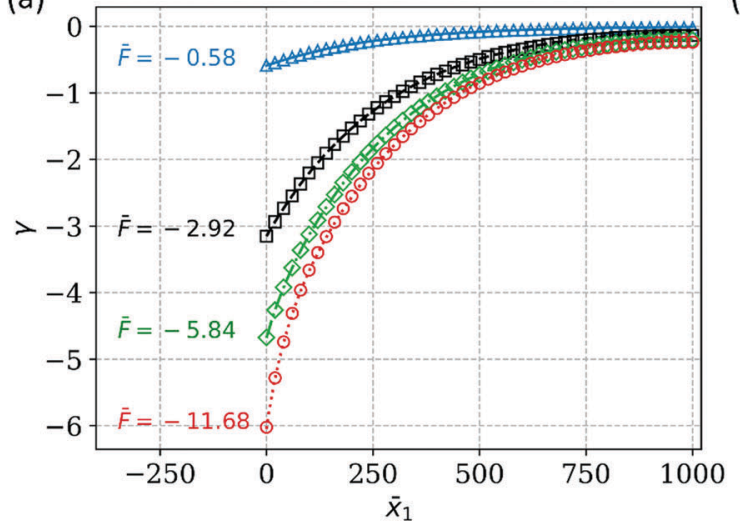

(b)

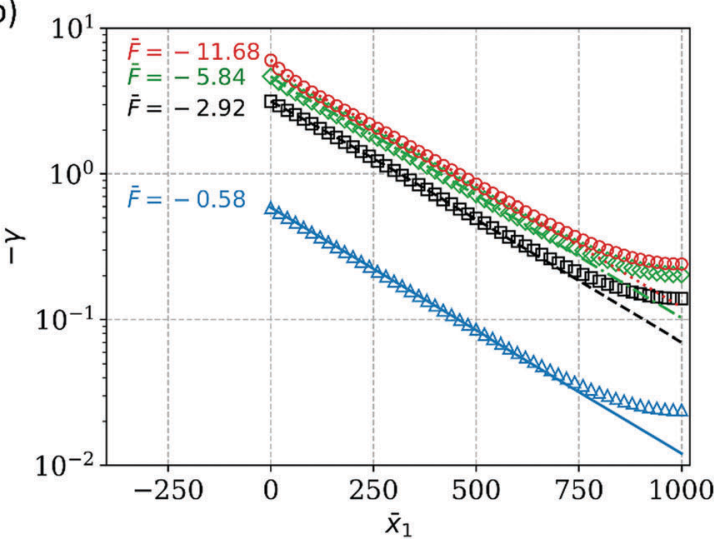

Fig. 6 Shear strain $\gamma$ plotted versus $\bar{x}_{1}$. Analytic solutions and FEM results are plotted as lines and symbols respectively: (a) linear-linear plot and (b) log-linear plot.
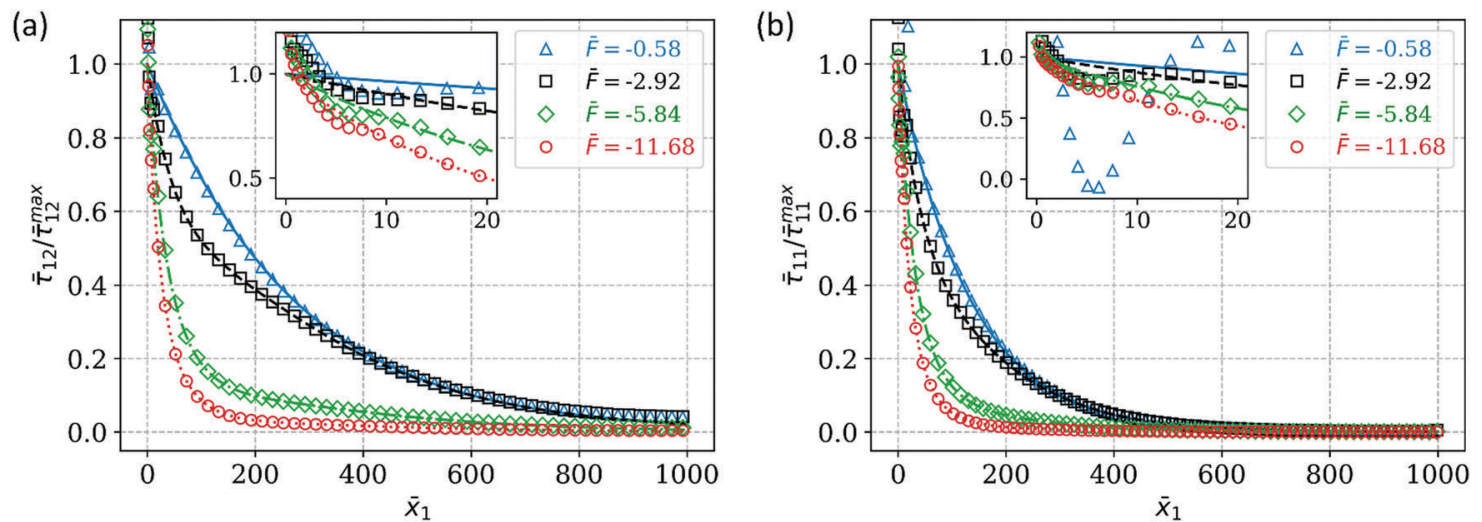

(c)

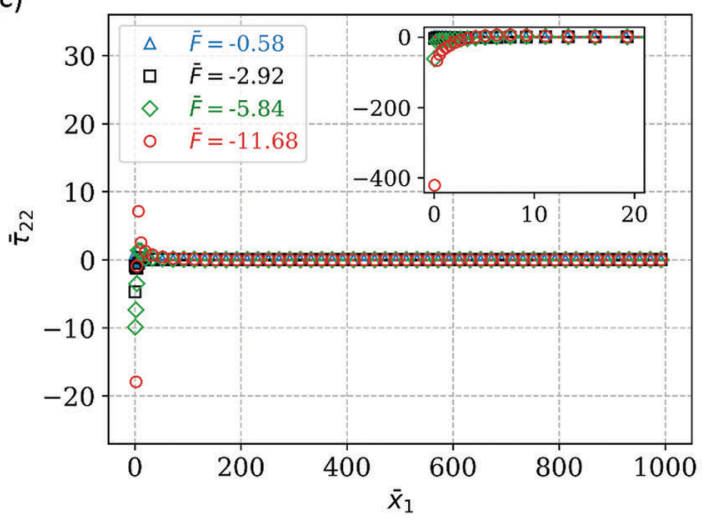

Fig. 7 True stress along the adhesive/substrate interface with different applied loads. Analytic solutions and FEM results are plotted as lines and symbols for different applied forces, i.e., $\bar{F}=-0.58,-2.92,-5.84$ and -11.68 respectively. The true stresses near the origin are plotted in the insets: (a) $\bar{\tau}_{12} / \bar{\tau}_{12}^{\max }$, (b) $\bar{\tau}_{11} / \bar{\tau}_{11}^{\max }$, and (c) $\bar{\tau}_{22}$.

model is surprisingly good at larger deformations, for example, for $\bar{F} \leq-2.92$, both the shear and normal stress agree reasonably well with the finite element result at distances of $\bar{x}_{1} \geq 3 h$. Finally, our finite element result in Fig. 7c confirms that the normal stress $\tau_{22}$ is indeed close to zero in region 2 .

Fig. S1 of the ESI $\dagger$ plots the FEM stresses on the adhesive/ backing interface. As expected, these stresses are practically the same in region 2 and agree well with our analytical model.

\subsection{Region 1: crack tip field}

In this section we focus on region 1 , where the stresses and strains are dominated by the presence of the interface crack. We study the stress field near the crack tip using the FEM. In particular, we plot the stresses along the adhesive/backing and adhesive/substrate interfaces. Fig. 8a shows that the normal stress $\bar{\tau}_{22}$ oscillates near the peel front due to the localized bending of the backing layer. Here we note that the results along 
both interfaces are plotted against the deformed configuration. Note that on the adhesive/substrate interface, there is no difference between deformed and undeformed coordinates, i.e., $x_{\alpha}=y_{\alpha}$, since the substrate is rigid. However, there are significant differences between these two configurations along the adhesive backing interface, as material points are unconstrained - this is why the horizontal axis in Fig. $8 \mathrm{~b}$ is labeled as $\bar{y}_{1}=y_{1} / h$. To highlight this difference, the profiles of the adhesive and backing are plotted in the bottom of Fig. $8 \mathrm{~b}$, and we pick four material points $\left(\bar{x}_{1}=-1,0,1\right.$, and $\left.2, \bar{x}_{2}=1\right)$ and their deformed coordinates are also indicated. Also, Fig. $8 \mathrm{~b}$ shows that when the deformation is large, the adhesive in front of the crack deforms severely. Due to the complexity of local geometry and effect of nonlinear elasticity, the stress fields in the adhesive layer near the adhesive/backing interface are very complicated, and this region is shaded in Fig. 8b. It is interesting to note that $\bar{\tau}_{22}$ along the adhesive/substrate interface changes rapidly from a pattern of 'tension-compressiontension' to 'compression-tension' as the applied force increases. In other words, for small peel forces, the material points on the adhesive/substrate interface that are closest to the crack tip are under tension. As the force increases, these material points are under compression. Recall that if the adhesive layers were to have zero thickness, linear elastic fracture mechanics would predict that an infinite compressive interfacial normal stress exists at the crack tip. However, our simulations suggested that, for an adhesive layer with finite thickness, the interfacial normal stress can actually be tensile, at least for small applied forces. Also, $\bar{\tau}_{22}$ retains the pattern of 'tension-compression-tension' on the adhesive/backing interface, irrespective of the peel force. In both cases, the amplitude of stress increases with the peel force.

It is interesting to compare our finite element result with Kaelble's prediction, which assumes small strains and uses a plate theory instead of solving the full elasticity equations. His result for the normal stress in the adhesive layer (see eqn (7) in his paper ${ }^{1}$ ), in the notation of this paper, is:

$$
\tau_{22}=-\beta^{2} F\left(2 h+h_{\mathrm{b}}\right) \mathrm{e}^{-\beta x_{1}}\left[\cos \beta x_{1}-\sin \beta x_{1}\right] / b,
$$

where

$$
\beta=\left[\frac{9 \mu}{E_{\mathrm{b}}{ }^{*} h_{\mathrm{b}}{ }^{3} h}\right]^{1 / 4} .
$$

This comparison is shown in Fig. 8a where Kaelble's results are plotted as dashed lines. Clearly, the linear theory fails to predict the stress. In addition, since the normal stress predicted by the linear theory is independent of $x_{2}$ and does not distinguish
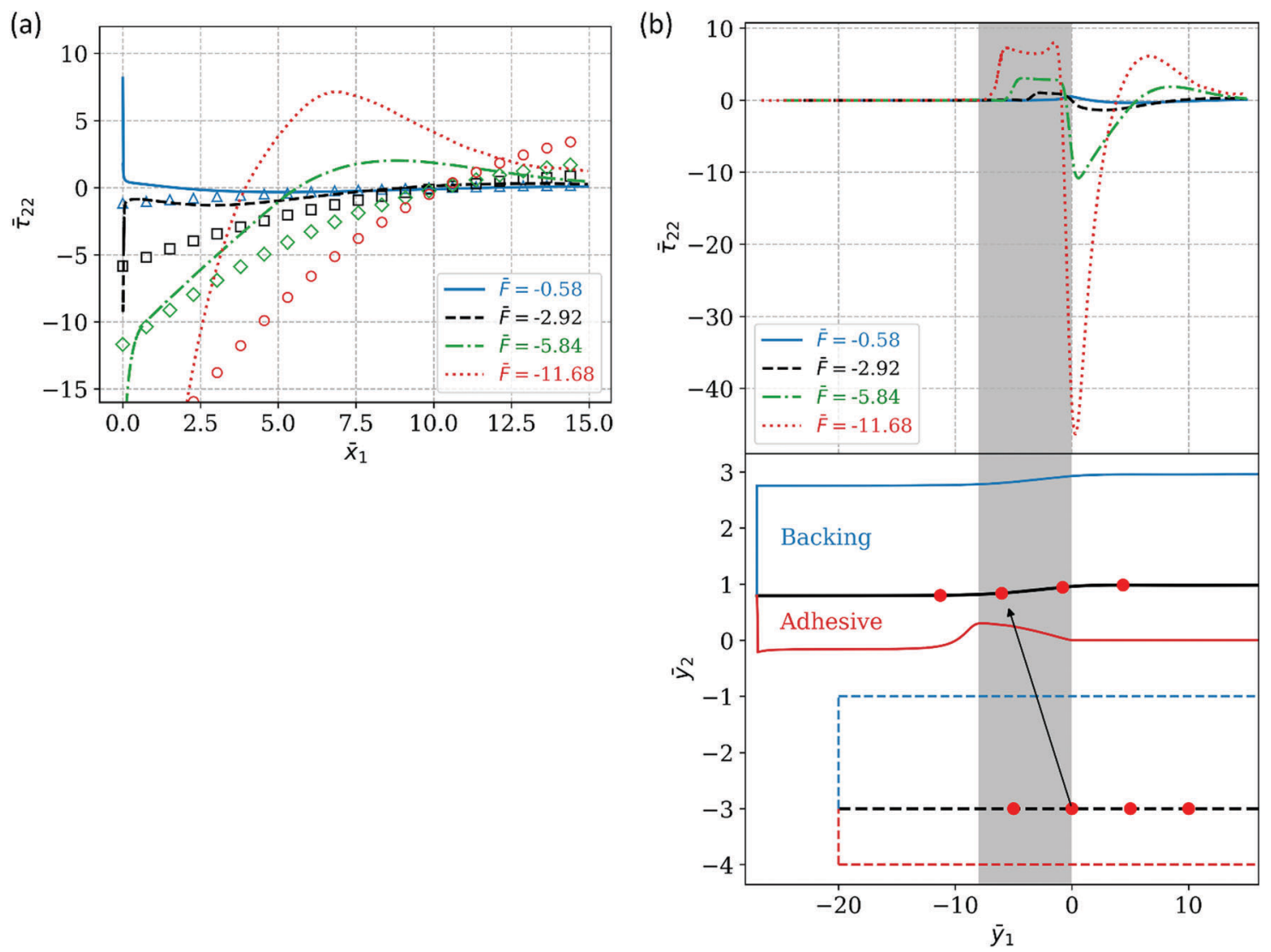

Fig. $8 \bar{\tau}_{22}$ along the adhesive/substrate and adhesive/backing interfaces with different applied forces. FEM results are plotted as solid, dashed, dasheddotted and dotted lines for $\bar{F}=-0.58,-2.92,-5.84$ and -11.68 respectively. (a) $\bar{\tau}_{22}$ along the adhesive/substrate interface. We also plot the linear theory predicted by Kaelble ${ }^{1}$ as symbols for comparison. (b) $\bar{\tau}_{22}$ along the adhesive/backing interface in the top image. Four material points (red circles in the bottom image) are plotted to indicate the deformation. 
between undeformed and deformed configurations, it cannot possibly capture the behavior as shown in Fig. 8b. Note that the characteristic length of decay of the normal stress $(1 / \beta)$ in Kaelble's theory is much smaller than the shear load transfer length $l_{\mathrm{LT}}$, since it depends on the ratio $\mu / E_{\mathrm{b}}{ }^{*}$ with an exponent $1 / 4$ instead of $1 / 2$.

Fig. 9a, b and 10a, b plot the normalized stress component $\bar{\tau}_{12}$ and $\bar{\tau}_{11}$ along the adhesive/substrate and adhesive/backing interfaces in region I respectively. The stresses due to $\bar{F}=-11.68$ are too large to fit in the same figure and we provide this information in the ESI. $\dagger$ Note that at higher peel forces these stresses behave differently on these interfaces. The normalized hydrostatic pressure $\bar{p} \equiv\left(\bar{\tau}_{11}+\bar{\tau}_{22}+\bar{\tau}_{33}\right) / 3$ along these interfaces are plotted in Fig. 11.

\section{Energy release rate}

As shown by Kendall, ${ }^{38,39}$ for a peel arm that is linearly elastic and bonded to a rigid substrate (with an infinitely thin adhesive), the energy release rate is exactly zero were it not for stretching of the peel arm. For our case the geometry is different: the thickness of the adhesive layer is comparable to that of the peel arm and part of the adhesive is bonded to the peel arm instead of the substrate.
Nevertheless, Kendall's conclusion is still true for our geometry. Indeed, in our geometry, crack growth by $\Delta a$ can be achieved by moving an element of length $\Delta a$ and height $h$ from $x_{1}=\infty$ to the load point. The amount of work done by the peel force as the crack advanced by $\Delta a$ is $(F / b) \varepsilon_{\mathrm{p}} \Delta a=\frac{(F / b)^{2}}{E_{\mathrm{b}}{ }^{*} h_{\mathrm{b}}} \Delta a$, where $\varepsilon_{\mathrm{p}}$ is the strain in the peel arm. Because the backing layer is linearly elastic and the adhesive in the peel arm carries no force, half of this work is used to increase the strain energy of this element as it moves from $x_{1}=\infty$ to the load point. Therefore, the energy that is available for crack growth is $\frac{(F / b)^{2}}{2 E_{\mathrm{b}}{ }^{*} h_{\mathrm{b}}} \Delta a$; so the energy release rate is exactly:

$$
G=\frac{(F / b)^{2}}{2 E_{\mathrm{b}}{ }^{*} h_{\mathrm{b}}} .
$$

Eqn (21) is valid for large deformation of the adhesive layer, as long as $L \gg l_{\mathrm{LT}}$. The critical peel force to initiate interface debonding $F_{\mathrm{c}}$ can be determined using energy balance: the energy release rate $G$ reaches the interfacial fracture energy $\Gamma$, i.e.,

$$
G=\frac{\left(F_{\mathrm{c}} / b\right)^{2}}{2 E_{\mathrm{b}}{ }^{*} h_{\mathrm{b}}}=\Gamma \Rightarrow F_{\mathrm{c}} / b=\sqrt{2 E_{\mathrm{b}}{ }^{*} h_{\mathrm{b}} \Gamma}
$$
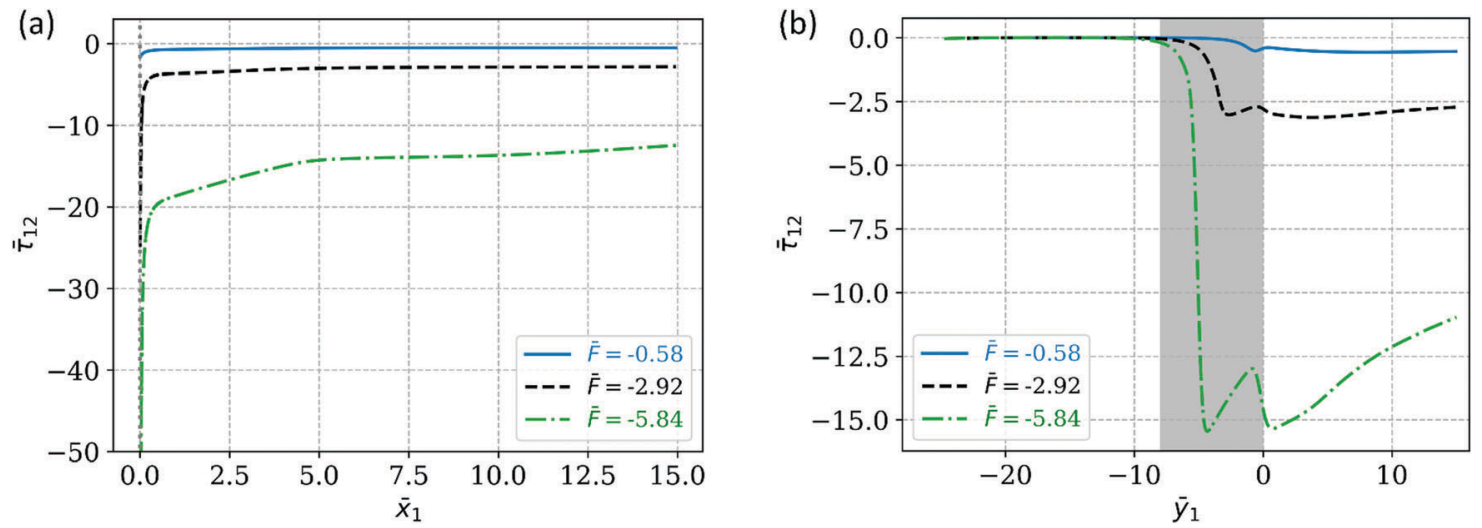

Fig. $9 \bar{\tau}_{12}$ along the adhesive/substrate and adhesive/backing with different applied forces. FEM results are plotted as solid, dashed and dashed-dotted lines for $\bar{F}=-0.58,-2.92$ and -5.84 respectively. (a) $\bar{\tau}_{12}$ along the adhesive/substrate interface. (b) $\bar{\tau}_{12}$ along the adhesive/backing interface.
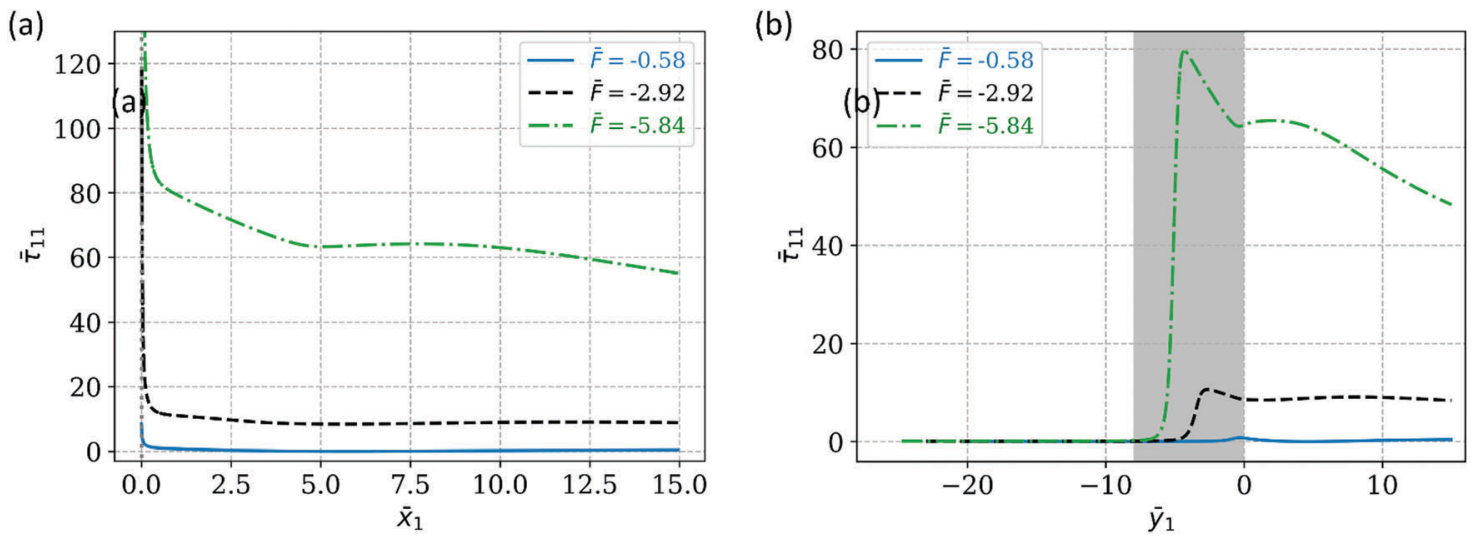

Fig. $10 \bar{\tau}_{11}$ along the adhesive/substrate and adhesive/backing with different applied forces. FEM results are plotted as solid, dashed and dashed-dotted lines for $\bar{F}=-0.58,-2.92$ and -5.84 respectively. (a) $\bar{\tau}_{11}$ along the adhesive/substrate interface. (b) $\bar{\tau}_{11}$ along the adhesive/backing interface. 
(a)

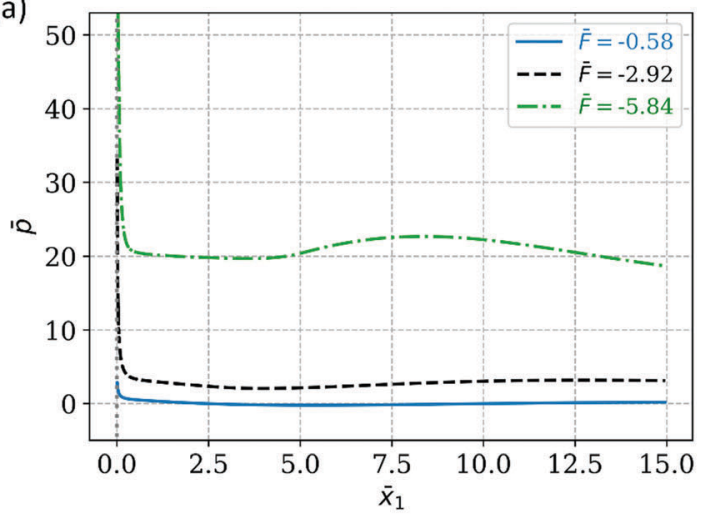

(b)

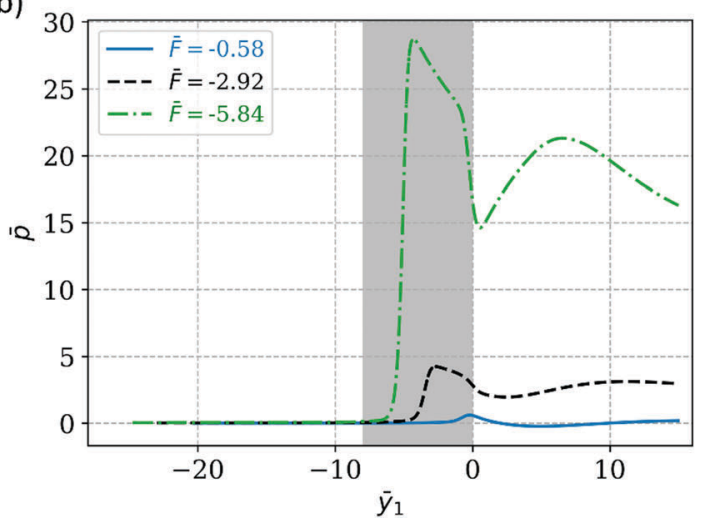

Fig. 11 Hydrostatic pressure $\bar{p}$ along the adhesive/substrate and adhesive/backing with different applied forces. FEM results are plotted as solid, dashed and dashed-dotted lines for $\bar{F}=-0.58,-2.92$ and -5.84 respectively. (a) $\bar{p}$ along the adhesive/substrate interface. (b) $\bar{p}$ along the adhesive/backing interface.

Note that the peel force needed to grow the interface crack can be very large, since the modulus of the backing layer is on the order of GPa. Here, one must distinguish between the energy release rate $G$ which is the amount of energy available for fracture and the interfacial fracture energy $\Gamma$. The former for this case is independent of the nonlinear elastic behavior of the adhesive and is controlled by the elasticity of the backing layer. The latter is a material property which depends on how the material fails near the crack tip. The local failure process is sensitive to the mechanical behavior of the adhesive, since the stress and strain states near the crack tip are very different for linear and nonlinear materials subjected to the same applied force. In other words, the interfacial fracture energy is controlled by the material behavior. Therefore, the critical peel force will be dependent on whether the material is linear or nonlinear. Note that we have assumed that the bond length $L$ is much longer than the load transfer length $l_{\mathrm{LT}}$, so that the energy release rate is independent of the bond length. This is certainly not the case for short bond lengths (i.e., $l_{\mathrm{LT}} \geq L$ ). For this case, the energy release rate is no longer given by (21); specifically, (21) underestimates the energy release rate. Indeed, when the crack grows (i.e., bond length decreases), the shear stress has to increase since the force $F / b$ is kept constant. This means that the energy release rate increases with crack extension (for smaller $L$ ), so unless $\Gamma$ increases with crack extension which is typically not the case, crack growth is unstable.

\section{Summary and discussion}

We present a large deformation analysis of the stress state in the adhesive layer of an adhesive tape subjected to a zero degree peel force. The adhesive layer is modeled as an incompressible hyper-elastic solid with a strain energy density function that depends only on the scalar invariant $I_{1}$ or the trace of the right Cauchy-Green tensor. Although our solution method does not depend on the form of the strain energy function, the solution is presented for a three-term Yeoh's model since this is the simplest material model that can capture the nonlinear elasticity of typical PSAs. Exact closed-form solutions are obtained for the in-plane stresses $\tau_{11}$ and $\tau_{12}$ in the adhesive layer in region 2. Surprisingly, even though the material is nonlinear and deformation is large, these stresses decay exponentially from the peel front with a characteristic distance equal to the load transfer length - which is an aspect of the linear theory. These exact solutions are in excellent agreement with our FEM. A surprising result is that for large shear deformation, the lateral true stress $\tau_{11}$ within the load transfer length is much larger than the shear stress, whereas in the linear theory this stress is assumed to be zero. As a result, the hydrostatic pressure in the adhesive layer is not small and cavities can nucleate and grow at distances far from the peel front. We compare our result with the linear theory which assumes small deformation and a linear elastic adhesive layer. Our results show that the linear theory fails to predict the peel force to sustain a given amount of deformation. For large deformation, the peel force is governed by the strain hardening behavior of the adhesive. Since our method in Section 2.3 can be used to produce an exact closed-form solution for any incompressible hyperelastic solids where the strain energy density function depends only on the trace of the right Cauchy-Green tensor, our result provides a path to study the zero degree peel test without the constraints and limitations imposed by linear elastic theory.

We also used a nonlinear FEM to study the 3D state of stress near the peel front. In this region (region 1), our analytic solution breaks down. In particular, the true normal stress $\tau_{22}$ can be very large and alternates between compression and tension. Although this result (an oscillatory state of normal stress) is predicted by the linear model of Kaelble, ${ }^{1}$ his result underestimates the stress near the peel front. This is due to the fact that Kaelble uses a plate model which cannot capture the 3D state of stress at the crack tip. In addition, his model assumes that the adhesive is linearly elastic, whereas a nonlinear elasticity model can account for stress increase due to strain hardening. More importantly, the plate model predicts that the normal and shear stresses are independent of $x_{2}$, whereas the stresses on the backing/adhesive interface and the adhesive/substrate interface are different as shown by our result in Fig. 9 and 10. It is also interesting to note that at high peel forces, the material directly ahead of 
the peel front (along the adhesive/substrate interface) is under compression while for the small peel force it is under tension. This, together with the fact that the stress states are different on each interface, may have important consequences on determining at which interface the adhesive fails.

The result presented here is limited to the case of the infinite bond length $L$, that is, when the bond length is much longer than the load transfer length $l_{\mathrm{LT}}$. In practice, the bond length can often be comparable or smaller than the load transfer length. For these cases, crack growth can be unstable since the energy release rate is expected to increase with crack length (or decreasing bond length), as noted by Mojdehi et al. ${ }^{17}$ Our approach can be extended to study this case and these results will be presented in a future study. A more serious limitation of our analysis is that we have not accounted for the viscoelasticity of the adhesive. Indeed, at the instant when the force is applied, the adhesive will have a larger shear modulus (due to its entangled structure) than at longer times, when it relaxes to its plateau modulus (due to its chemical crosslinking). Viscous creep will then cause the load transfer length to increase with time. Thus, our analysis is strictly correct for short or long times. Specifically, for times much shorter than the characteristic relaxation time $\tau$ of the adhesive, the moduli in our model should be replaced with the short time moduli; for times much longer than $\tau$, the moduli in our model should be replaced with the plateau moduli. It is possible to include linear viscoelasticity in our model using a very crude approximation. Kaelble ${ }^{40}$ proposed that his elastic peel model can be extended to include linear viscoelasticity if the elastic shear modulus of the adhesive is replaced by its relaxation modulus. We apply this idea to our nonlinear model, that is, we replace $\mu$ in (17) by the shear relaxation modulus $\mu(t)$ while $C_{2} / \mu(t)$ and $C_{3} / \mu(t)$ are assumed to be material constants independent of time and $\mu(t)$ is assumed to be independent of strain. In particular, the load transfer length will now be time dependent, i.e.,

$$
l_{\mathrm{LT}} \equiv \sqrt{E_{\mathrm{b}}{ }^{*} h_{\mathrm{b}} h / \mu(t)} .
$$

Certainly the approximation given by (23) is valid for short and long times. In the ESI, $\dagger$ we use a simple viscoelastic model with one relaxation time to check this idea and find that the shear strain distribution at intermediate times is predicted quite well by this approximation. Of course, the relaxation behavior of real adhesives is much more complex and will not be captured well by a linear viscoelastic model with a single relaxation time. The role of viscoelastic behavior in the peel test will be the subject of a future study.

However for highly deformed adhesives in shear, our nonlinear elastic solution provides realistic stress distributions and kinematics in the adhesive for a given applied load. This result can then be used to predict much more accurately the locus of failure in the adhesive, which is a long-standing problem in this geometry. As has been shown, a linear solution will predict the stresses incorrectly and will give predictions that are inconsistent with experiments. Furthermore, many changes in the chemistry of the adhesive and in particular crosslinking will affect only non-linear properties and not linear properties. A linear model will fail to capture the effect of those differences and has no chance at all to predict failure correctly.

\section{Conflicts of interest}

There are no conflicts to declare.

\section{Acknowledgements}

C. Y. Hui acknowledges the support by the National Science Foundation under Grant No. CMMI-1537087. Helen Minsky's postdoctoral fellowship is supported by $3 \mathrm{M}$ Company. We are also grateful to anonymous reviewers for their helpful comments.

\section{References}

1 D. H. Kaelble, Trans. Soc. Rheol., 1960, 4, 45-73.

2 H. Lakrout, C. Creton, D. Ahn and K. R. Shull, Macromolecules, 2001, 34, 7448-7458.

3 A. Zosel, Int. J. Adhes. Adhes., 1998, 18, 265-271.

4 C. Creton and H. Lakrout, J. Polym. Sci., Part B: Polym. Phys., 2000, 38, 965-979.

5 H. Lakrout, P. Sergot and C. Creton, J. Adhes., 1999, 69, 307-359.

6 A. J. Crosby, K. R. Shull, H. Lakrout and C. Creton, J. Appl. Phys., 2000, 88, 2956-2966.

7 Y. Y. Lin, C.-Y. Hui and H. D. Conway, J. Polym. Sci., Part B: Polym. Phys., 2000, 38, 2769-2784.

8 A. N. Gent and C. Wang, J. Mater. Sci., 1991, 26, 3392-3395.

9 A. N. Gent, J. Polym. Sci., Part A-2, 1971, 9, 283-294.

10 A. N. Gent and R. P. Petrich, Proc. R. Soc. A, 1969, 310, 433-448.

11 P. S. T. Council, Test methods for pressure sensitive adhesive tapes, Pressure Sensitive Tape Council, 1994.

12 A. Zosel, J. Adhes., 1994, 44, 1-16.

13 F. Sosson, A. Chateauminois and C. Creton, J. Polym. Sci., Part B: Polym. Phys., 2005, 43, 3316-3330.

14 S. M. Xia, L. Ponson, G. Ravichandran and K. Bhattacharya, J. Mech. Phys. Solids, 2013, 61, 838-851.

15 K. Kendall, J. Phys. D: Appl. Phys., 1975, 8, 512-522.

16 H. L. Cox, Br. J. Appl. Phys., 1952, 3, 72-79.

17 A. R. Mojdehi, D. P. Holmes and D. A. Dillard, Int. J. Solids Struct., 2017, 124, 125-134.

18 S. Ponce, J. Bico and B. Roman, Soft Matter, 2015, 11, 9281-9290.

19 R. R. Collino, N. R. Philips, M. N. Rossol, R. M. McMeeking and M. R. Begley, J. R. Soc., Interface, 2014, 11, 20140453.

20 T. Cohen, C. U. Chan and L. Mahadevan, Soft Matter, 2018, 14, 1771-1779.

21 A. J. Crosby and K. R. Shull, J. Polym. Sci., Part B: Polym. Phys., 1999, 37, 3455-3472.

22 S. Vajpayee, C.-Y. Hui and A. Jagota, Langmuir, 2008, 24, 9401-9409.

23 A. J. Kinloch, Adhesion and adhesives: science and technology, Springer Science \& Business Media, 2012. 
24 B. Lawn, Fracture of Brittle Solids, Cambridge University Press, Cambridge, 2nd edn, 1993.

25 M. D. Bartlett, A. B. Croll, D. R. King, B. M. Paret, D. J. Irschick and A. J. Crosby, Adv. Mater., 2012, 24, 1078-1083.

26 C. Creton, MRS Bull., 2003, 28, 434-439.

27 C. Creton, A. Roos and A. Chiche, Effect of the diblock content on the adhesive and deformation properties of PSAs based on styrenic block copolymers, Wiley-VCH, Weinheim, 2005.

28 C. Creton, G. Hu, F. Deplace, L. Morgret and K. R. Shull, Macromolecules, 2009, 42, 7605-7615.

29 Z. Suo and J. W. Hutchinson, Int. J. Fract., 1990, 43, 1-18.

30 M. D. Thouless and Q. D. Yang, Int. J. Adhes. Adhes., 2008, 28, 176-184.

31 R. Villey, P.-P. Cortet, C. Creton and M. Ciccotti, Int. J. Fract., 2017, 204, 175-190.
32 J. Chopin, R. Villey, D. Yarusso, E. Barthel, C. Creton and M. Ciccotti, Macromolecules, 2018, 51, 8605-8610.

33 A. Bellamine, E. Degrandi, M. Gerst, R. Stark, C. Beyers and C. Creton, Macromol. Mater. Eng., 2011, 296, 31-41.

34 A. Lindner, B. Lestriez, S. Mariot, C. Creton, T. Maevis, B. Lühmann and R. Brummer, J. Adhes., 2006, 82, 267-310.

35 F. Tanguy, M. Nicoli, A. Lindner and C. Creton, Eur. Phys. J. E: Soft Matter Biol. Phys., 2014, 37, 3.

36 F. Deplace, C. Carelli, S. Mariot, H. Retsos, A. Chateauminois, K. Ouzineb and C. Creton, J. Adhes., 2009, 85, 18-54.

37 M. Abramowitz and I. A. Stegun, Handbook of mathematical functions: with formulas, graphs, and mathematical tables, Courier Corporation, 1964, vol. 55.

38 K. Kendall, J. Phys. D: Appl. Phys., 1971, 4, 1186-1195.

39 K. Kendall, J. Phys. D: Appl. Phys., 1975, 8, 1449-1452.

40 D. H. Kaelble, J. Adhes., 1992, 37, 205-214. 